\title{
De novo biosynthesis of para-nitro-L-phenylalanine in Escherichia coli
}

3 Neil D. Butler, Sabyasachi Sen, Minwei Lin, and Aditya M. Kunjapur*

4 Department of Chemical \& Biomolecular Engineering, University of Delaware, Newark, DE 5 19716, United States

$6 \quad *$ Corresponding Author

7 Aditya Kunjapur - Department of Chemical \& Biomolecular Engineering, University of 8 Delaware, Newark, DE 19716, United States; orcid.org/0000-0001-6869-9530

$9 \quad$ Email: kunjapur@udel.edu

Highlights

- Para-nitro-L-phenylalanine (pN-Phe) is a valuable small molecule for its applications in genetic code expansion.

- We establish de novo biosynthesis of $\mathrm{pN}-\mathrm{Phe}$ from glucose in E. coli, which is also the first example of a de novo pathway design for an unnatural but commonly used non-standard amino acid.

- We show the first use of an $N$-oxygenase enzyme in the de novo synthesis of a nitroaromatic product.

- Screening of natural $N$-oxygenases and strain engineering resulted in final $\mathrm{pN}$-Phe titers of $820 \pm 130 \mu \mathrm{M}$ in shake flask experiments with rich defined media.

Keywords

$31 \mathrm{~N}$-oxygenase, para-nitro-L-phenylalanine, nitrophenylalanine, nitroaromatic, de novo 32 biosynthesis, non-standard amino acids, genetic code expansion 


\section{Abstract}

Nitroaromatic functional groups can impart valuable properties to chemicals and to biological macromolecules including polypeptides. Para-nitro-L-phenylalanine (pN-Phe) is a nitroaromatic amino acid with uses including immune stimulation and fluorescence quenching. As the chemical synthesis of $\mathrm{pN}$-Phe does not follow green chemistry principles and impedes provision of $\mathrm{pN}-\mathrm{Phe}$ to engineered bacterial cells in some contexts, we sought to design a de novo biosynthetic pathway for pN-Phe in Escherichia coli. To generate the nitro chemical functional group, we identified natural diiron monooxygenases with measurable in vitro and in vivo activity on envisioned aminecontaining precursors of para-amino-L-phenylalanine (pA-Phe) and para-aminophenylpyruvate. By expressing one of these $N$-oxygenase genes together with previously characterized genes for the biosynthesis of $\mathrm{pA}-\mathrm{Phe}$, we achieved the synthesis of $\mathrm{pN}$-Phe from glucose. Through further optimization of the chassis, plasmid constructs, and media conditions, we were able to improve the selectivity of $\mathrm{pN}$-Phe biosynthesis, resulting in a maximum titer of $819 \mu \mathrm{M}$ in rich defined media under shake-flask conditions. These results provide a foundation for the biosynthesis of related nitroaromatic chemicals and for downstream biological applications that could utilize $\mathrm{pN}$ Phe as a building block.

\section{Introduction}

Nitro-group containing compounds, and particularly nitroaromatics, are an important class of chemicals with industrial applications as herbicides, energetic materials, and pharmaceuticals (Booth, 2000). In biological environments, the polarity of nitro-groups generates unique binding interactions with protein receptors and other molecules which enables application of nitroaromatics as antibiotics, antiparasitics, and chemotherapeutics (Nepali et al., 2019; Strauss, 1979). Specifically, the strong $\pi$ electron delocalizing properties of the nitro group within aromatic 
systems can enhance intermolecular interactions including arene-arene and aryl hydrogen anion

57 bonding interactions (Bryantsev and Hay, 2005; Lewis et al., 2012; Shorter, 2009). While proteins could also benefit from the installation of these properties at specific sites, none of the twenty

59 standard amino acids exhibit similar levels of electron-withdrawing potential. For this and other reasons, researchers have been interested in using genetic code expansion technology to

61 incorporate nitroaromatic non-standard amino acids (nsAAs) within proteins (Arbely et al., 2012;

62 Chen et al., 2009; Deiters et al., 2006; Gautier et al., 2010; Hancock et al., 2010; Hemphill et al.,

63 2013; Lemke et al., 2007; Neumann et al., 2008; Nguyen et al., 2014; Peters et al., 2009; Stokes et

64 al., 2009; Tsao et al., 2006; Wang et al., 2013, 2010; Welegedara et al., 2018; Wilkins et al., 2010;

65 Wu et al., 2004; Yanagisawa et al., 2012). The nitroaromatic non-standard amino acid para-nitro-

66 L-phenylalanine ( $\mathrm{pN}-\mathrm{Phe}$ ) in particular has been utilized in several diverse applications including

67 as a peptide distance marker (Tsao et al., 2006), a breaker of immune self-tolerance (Gauba et al.,

68 2011; Grünewald et al., 2009, 2008), a nitroreductase activity enhancer (Jackson et al., 2006), and an internal protein IR probe (Smith et al., 2011).

To date, the reliance on the supplementation of chemically synthesized $\mathrm{pN}$-Phe has constrained the performance of cells that are engineered to harness this building block and limited their potential end-applications. Microbial biosynthesis of nsAAs presents a promising alternative

73 because it eliminates external supplementation requirements, circumvents cellular uptake

74 limitations, and lowers toxicity through on-demand synthesis and incorporation (Dickey et al., 75 2021; Völler and Budisa, 2017). Moreover, the ability to biosynthesize nsAAs from simple carbon 76 sources should allow microbes to perform augmented protein functions autonomously in 77 environmental and therapeutic contexts where provision is not possible (Parker and Kunjapur, 78 2020). Pioneering work has achieved biosynthesis of a limited number of nsAAs, including para- 
amino-L-phenylalanine (pA-Phe) (Chen et al., 2018; Mehl et al., 2003), L-dihydroxyphenylalanine (Kim et al., 2018), 4-nitro-L-tryptophan (Zuo and Ding, 2019), 5-hydroxytryptophan (Chen et al., 2020), and propargylglycine (Marchand et al., 2019). While significant advancements, these nsAAs are found in nature and were produced in model microbes by transplanting naturally occurring and highly specific pathways. To our knowledge, $\mathrm{pN}-\mathrm{Phe}$ has not been found in nature (Parry et al., 2011; Waldman et al., 2017) and would thus require de novo pathway design for its biosynthesis.

Design of metabolic syntheses for nitroaromatic products such as $\mathrm{pN}$-Phe can also provide an alternative to the harsh acidic reaction conditions and poor regioselectivity of traditional chemical nitration (Badgujar et al., 2016; Liu et al., 2009; Yan and Yang, 2013). To construct a metabolic pathway that achieves biosynthesis of $\mathrm{pN}-\mathrm{Phe}$, we anticipated that nitro group formation could proceed from aromatic metabolites via one of the two major characterized methods of enzymatic nitro synthesis: biocatalytic direct nitration or amine oxidation (Sulzbach and Kunjapur, 2020; Winkler and Hertweck, 2007). Biocatalytic direct nitration is a process dependent on two enzymatic steps: (1) the cytochrome P450-mediated nitration of aromatic rings using $\mathrm{O} 2$ and nitric oxide (NO) and (2) the synthesis of reactive NO from the activity of nitric oxide synthases (NOS) (Caranto, 2019). To date, only two P450/NOS pairs for aromatic direct nitration have been identified, and neither has demonstrated activity to nitrate phenylalanine (Barry et al., 2012; Dodani et al., 2016; Tomita et al., 2017; Zuo et al., 2017). Amine oxidation, on the other hand, is a promising route for $\mathrm{pN}-\mathrm{Phe}$ synthesis for two reasons. First, the metabolic synthesis of the amine precursor, pA-Phe has been demonstrated from multiple natural gene clusters (commonly referred to as papABC) (Chen et al., 2018; Masuo et al., 2016; Mehl et al., 2003). Second, a promising amine oxidizing enzyme, or $\mathrm{N}$-oxygenase (ObaC, otherwise referred to in the literature as ObiL), 
102

103

104

105

106

107

108

109

110

111

112

113

114

115

116

117

118

119

120

121

122

was recently discovered in Pseudomonas fluorescens in the biosynthetic gene cluster for the antibiotic obafluorin. Preliminary and indirect evidence suggests that this $N$-oxygenase has native activity on para-aminophenylpyruvate (pA-Pyr), the immediate precursor to pA-Phe in metabolic synthesis (Schaffer et al., 2017; Scott et al., 2017). Despite exploration of several $N$-oxygenases like ObaC with diiron monooxygenase activity, namely AurF (Chanco et al., 2014) (with a native substrate of para-aminobenzoic acid) and CmlI (Tsutsumi et al., 2018) (with a native substrate of the amine precursor to chloramphenicol) as potential in vitro catalysts for nitroaromatic product synthesis, thus far, no $N$-oxygenases have been used in vivo for de novo biosynthetic cascades despite their promise and demonstrated activity in E. coli.

Here, we constructed and tested a de novo biosynthetic pathway in Escherichia coli based on the following enzymes (Fig. 1): (i) Three enzymes from the chloramphenicol biosynthesis pathway in Streptomyces venezuelae that generate pA-Phe from chorismate; (ii) the putative $N$-oxygenase ObaC; (iii) the ability of native E. coli aminotransferases to convert keto acids to amino acids (Hayashi et al., 1993; Onuffer et al., 1995). Upon confirmation of de novo pN-Phe biosynthesis, we transferred the proposed enzymes to a chorismate overproducing strain of E. coli which further improved product titers. We then performed sequence similarity analysis to identify $N$-oxygenase enzymes related to those previously characterized and identified a candidate (NO16) that achieves higher conversion of pA-Phe than ObaC. Integration of this $N$-oxygenase improved pN-Phe titers with maximum titer observed in MOPS-EZ rich defined media. 


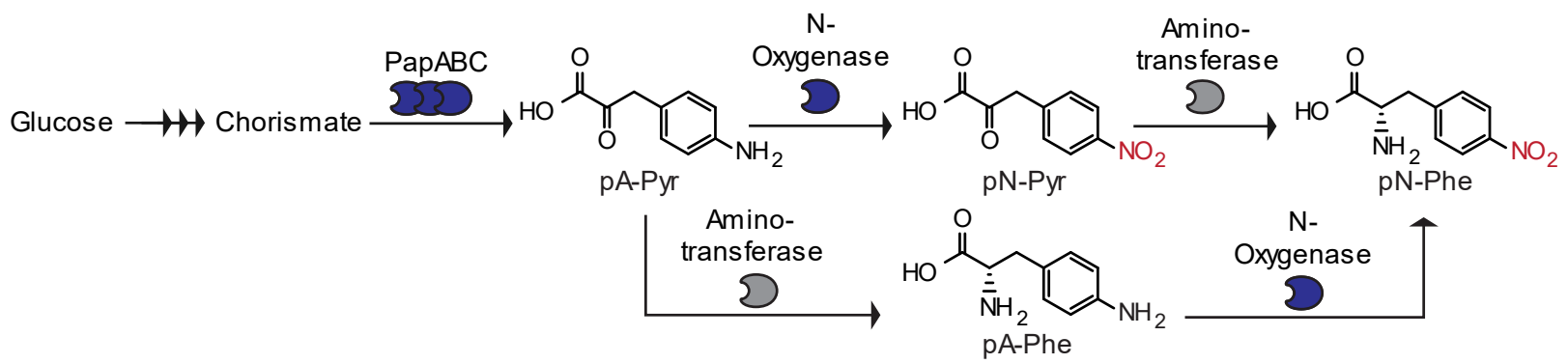

Figure 1. A graphical overview of the proposed de novo biosynthesis pathway for para-nitro-L-

126 phenylalanine ( $\mathrm{pN}-\mathrm{Phe}$ ) with exogenous enzymes shown in blue and endogenous enzymes shown

127 in gray. PapA, 4-amino-4-deoxychorismate synthase; PapB, 4-amino-4-deoxychorismate mutase;

128 PapC, 4-amino- 4-deoxyprephenate dehydrogenase; pA-Pyr, para-aminophenylpyruvate; pN-Pyr, 129 para-nitrophenylpyruvate; pA-Phe, para- amino-L-phenylalanine. 
131

132

\section{Materials and Methods}

2.1. Strains and plasmids.

Molecular cloning and vector propagation were performed in DH5 $\alpha$. Polymerase chain reaction (PCR) based DNA replication was performed using KOD XTREME Hot Start Polymerase for plasmid backbones or using KOD Hot Start Polymerase otherwise. Cloning was performed using Gibson Assembly. Genes were purchased as G-Blocks or gene fragments from Integrated DNA Technologies (IDT) or Twist Bioscience and were optimized for E. coli K12 using the IDT Codon Optimization Tool. The plasmids pOSIP-TH (Addgene plasmid \# 45978; http://n2t.net/addgene:4598; RRID:Addgene_45988) and pE-FLP (Addgene plasmid \# 45978; http://n2t.net/addgene:45978 ; RRID:Addgene_45978) were gifts from Drew Endy \& Keith Shearwin (Cui and Shearwin, 2017). The papABC operon was kindly provided by Professor Ryan Mehl of Oregon State University in plasmid pLASC-lppPW. The pORTMAGE-EC1 recombineering plasmid was kindly provided by Timothy Wannier of the University of Ottawa (Wannier et al., 2020).

The chorismate overproducer strain was derived from a commercially available phenylalanine overproducer E. coli strain (NST37, ATCC \#31882). To enable the compatibility of this strain with T7-promoter systems, the $4521 \mathrm{bp}$ region of the phage $\mathrm{T} 7$ genome that is responsible for $\mathrm{T} 7$ polymerase functionality (lacI, lacZ, and T7 RNA polymerase) was genomically integrated using one-step clonetegration with the pOSIP-TH plasmid. Following plasmid assembly, NST37 was transformed with the clonetegration plasmid, and the integration of the region for $\mathrm{T} 7$ polymerase activity (DE3) was confirmed via selection on LB-agar plates containing $9 \mu \mathrm{g} / \mathrm{mL}$ tetracycline. Following Sanger sequencing-based confirmation of genetic incorporation, genomic tetracycline resistance was removed using pE-FLP. Then, using multiplexed automated genome engineering 
154 (MAGE) with the pORTMAGE-EC1 recombineering plasmid, two stop codons were introduced

155 into the genomic sequence for the chorismate mutase/prephenate dehydratase PheA at positions

15610 and 12 to serve as a translational knockout. Curing of the pORTMAGE-EC1 plasmid following

157 Sanger sequencing confirmation of genomic knockouts produced the chorismate overproducer 158 strain (NST37 (DE3) $\Delta p h e A)$.

\subsection{Chemicals}

The following compounds were purchased from MilliporeSigma: hydrogen peroxide,

161 kanamycin sulfate, chloramphenicol, carbenicillin disodium, dimethyl sulfoxide (DMSO), 162 potassium phosphate dibasic, potassium phosphate monobasic, magnesium sulfate, calcium 163 chloride dihydrate, imidazole, glycerol, M9 salts, sodium dodecyl sulfate, lithium hydroxide, boric 164 acid, Tris base, glycine, HEPES, and KOD XTREME Hot Start and KOD Hot Start polymerases. 165 pN-Phe and D-glucose were purchased from TCI America. pA-Phe, methanol, agarose, Laemmli 166 SDS sample reducing buffer, and ethanol were purchased from Alfa Aesar. pA-Pyr and pN-Pyr 167 were purchased from abcr GmbH. Anhydrotetracycline (aTc) and isopropyl $\beta$-D-1168 thioglactopyranoside (IPTG) were purchased from Cayman Chemical. Acetonitrile, sodium 169 chloride, LB Broth powder (Lennox), LB Agar powder (Lennox), were purchased from Fisher 170 Chemical. Trace Elements A was purchased from Corning. L-Arabinose was purchased from 171 VWR. A MOPS EZ rich defined medium kit was purchased from Teknova. Taq DNA ligase was 172 purchased from GoldBio. Phusion DNA polymerase and T5 exonuclease were purchased from 173 New England BioLabs (NEB). Sybr Safe DNA gel stain and BenchMark ${ }^{\mathrm{TM}}$ His-tagged Protein 174 Standard were purchased from Invitrogen. HRP-conjugated 6*His His-Tag Mouse McAB was 175 obtained from Proteintech. Oligonucleotides were purchases from IDT. Gene fragments were 
176 purchased from Twist Bioscience with the exception of ObaC-ObaD which was purchased from

177 IDT.

\section{$178 \quad$ 2.3.Culture conditions}

179 Cultures for general culturing, for experiments in Figures 2A, 2B, 3, 5B S4, and S6, and $N$ oxygenase protein overexpression were grown in LB-Lennox medium (LBL: $10 \mathrm{~g} / \mathrm{L}$ bacto tryptone, $5 \mathrm{~g} / \mathrm{L}$ sodium chloride, $5 \mathrm{~g} / \mathrm{L}$ yeast extract). Cultures to demonstrate de novo $\mathrm{pN}-\mathrm{Phe}$ synthesis were grown in either LB-Lennox-glucose medium (LBL with $1.5 \%$ glucose (wt/vol)), M9-glucose minimal media (Kunjapur et al., 2016) with Corning® Trace Elements A $(1.60 \mu \mathrm{g} / \mathrm{mL}$ $\mathrm{CuSO}_{4} \cdot 5 \mathrm{H}_{2} \mathrm{O}, 863.00 \mu \mathrm{g} / \mathrm{mL} \mathrm{ZnSO}_{4} \cdot 7 \mathrm{H}_{2} \mathrm{O}, 17.30 \mu \mathrm{g} / \mathrm{mL}$ Selenite $\bullet 2 \mathrm{Na}, 1155.10 \mu \mathrm{g} / \mathrm{mL}$ ferric citrate) and $1.5 \%$ glucose (wt/vol), or MOPS EZ rich defined media (Teknova M2105) with 1.5\% glucose (wt/vol). For cultures of NST37(DE3) $4 p h e A$ strains that were grown in M9-glucose minimal media, $0.04 \mathrm{mg} / \mathrm{ml}$ L-phenylalanine, $0.04 \mathrm{mg} / \mathrm{ml}$ L-tyrosine, and $0.04 \mathrm{mg} / \mathrm{mL} \mathrm{L-}$ tryptophan were added to the media to ensure growth.

For stability testing, a culture of $E$. coli K12 MG1655 (DE3) was inoculated from a frozen stock and grown to confluence overnight in $5 \mathrm{~mL}$ of LBL media. Confluent overnight cultures were then used to inoculate experimental cultures in $300 \mu \mathrm{L}$ volumes in a 96-deep-well plate 192 (Thermo Scientific ${ }^{\mathrm{TM}} 260251$ ) at 100x dilution. Cultures were supplemented with $0.5 \mathrm{mM}$ of 193 heterologous metabolites (pA-Phe, pA-Pyr, pN-Pyr, pN-Phe), with pN-Pyr requiring an addition 194 of $15 \mu \mathrm{L}$ of DMSO ( 5\% final concentration) for solubility. Cultures were incubated at $37^{\circ} \mathrm{C}$ with 195 shaking at $1000 \mathrm{RPM}$ and an orbital radius of $3 \mathrm{~mm}$. Compounds were quantified from the 196 extracellular broth over a $24 \mathrm{~h}$ period using HPLC.

197 For toxicity testing, cultures were similarly prepared with confluent overnight cultures of 198 MG1655 (DE3) used to inoculate experimental cultures at 100x dilution in $200 \mu \mathrm{L}$ volumes in a 
Greiner clear bottom 96 well plate (Greiner 655090) in LBL media. Cultures were supplemented with $1 \mathrm{mM}$ of heterologous metabolite and 5\% DMSO for metabolite solubility and grown for 24

201 $\mathrm{h}$ in a Spectramax i3x plate reader with medium plate shaking at $37^{\circ} \mathrm{C}$ with absorbance readings at $600 \mathrm{~nm}$ taken every $5 \mathrm{~min}$ to calculate doubling time and growth rate. prepared with inoculation of $300 \mu \mathrm{L}$ volumes in a 96-deep-well plate with appropriate antibiotic added to maintain plasmids (34 $\mu \mathrm{g} / \mathrm{mL}$ chloramphenicol $(\mathrm{Cm}), 50 \mu \mathrm{g} / \mathrm{mL}$ kanamycin (Kan), 50 with shaking at 1000 RPM and an orbital radius of $3 \mathrm{~mm}$ until an $\mathrm{OD}_{600}$ of 0.5-0.8 was reached. measured via supernatant sampling and submission to HPLC. grown at $37{ }^{\circ} \mathrm{C}$ at $250 \mathrm{RPM}$. Expression vectors were fully induced at $\mathrm{OD}_{600} 0.5-0.8$, and then

217 were cultured at $30^{\circ} \mathrm{C}$. Synthesis of metabolites was quantified via supernatant sampling over 24

$218 \mathrm{~h}$ and analysis by HPLC. Compound confirmation was performed by UPLC-MS. 
222 or NB38) was inoculated from frozen stocks and grown to confluence overnight in $5 \mathrm{~mL}$ LBL

223 containing kanamycin. Confluent cultures were used to inoculate $400 \mathrm{~mL}$ of experimental culture

224 of LBL supplemented with kanamycin. The culture was incubated at $37^{\circ} \mathrm{C}$ until an $\mathrm{OD}_{600}$ of $0.5-$

2250.8 was reached while in a shaking incubator at 250 RPM. $N$-oxygenase expression was induced

226 by addition of anhydrotetracycline $(0.2 \mathrm{nM})$ and cultures were incubated at $30^{\circ} \mathrm{C}$ for $5 \mathrm{~h}$. Cultures

227 were then grown at $20{ }^{\circ} \mathrm{C}$ for an additional $18 \mathrm{~h}$. Cells were centrifuged using an Avanti J-15R

228 refrigerated Beckman Coulter centrifuge at $4{ }^{\circ} \mathrm{C}$ at $4,000 \mathrm{~g}$ for $15 \mathrm{~min}$. Supernatant was then

229 aspirated and pellets were resuspended in $8 \mathrm{~mL}$ of lysis buffer (25 mM HEPES, $10 \mathrm{mM}$ imidazole,

$230300 \mathrm{mM} \mathrm{NaCl}, 10 \%$ glycerol, $\mathrm{pH}$ 7.4) and disrupted via sonication using a QSonica Q125 sonicator

231 with cycles of $5 \mathrm{~s}$ at $75 \%$ amplitude and $10 \mathrm{~s}$ off for 5 minutes. The lysate was distributed into

232 microcentrifuge tubes and centrifuged for $1 \mathrm{~h}$ at $18,213 \mathrm{Xg}$ at $4{ }^{\circ} \mathrm{C}$. The protein-containing

233 supernatant was then removed and loaded into a HisTrap Ni-NTA column using an ÄKTA Pure

234 GE FPLC system. Protein was washed with 3 column volumes (CV) at $60 \mathrm{mM}$ imidazole and 4

$235 \mathrm{CV}$ at $90 \mathrm{mM}$ imidazole. $N$-oxygenase was eluted in $250 \mathrm{mM}$ imidazole in $1.5 \mathrm{~mL}$ fractions.

236 Selected fractions were denatured in Lamelli SDS reducing sample buffer $(62.5 \mathrm{mM}$ Tris- $\mathrm{HCl}$,

$237 \quad 1.5 \%$ SDS, $8.3 \%$ glycerol, $1.5 \%$ beta-mercaptoethanol, $0.005 \%$ bromophenol blue) for 10 minutes

238 at $95{ }^{\circ} \mathrm{C}$ and subsequently run on an SDS-PAGE gel with a Thermo Scientific PageRuler ${ }^{\mathrm{TM}}$

239 Prestained Plus ladder to identify protein containing fractions and confirm their size. The $N$ -

240 oxygenase containing fractions were combined applied to an Amicon column (10 kDa MWCO)

241 and the buffer was diluted 1,000x into a $20 \mathrm{mM}$ Tris $\mathrm{pH} 8.0,5 \%$ glycerol buffer.

242

\section{5. $N$-oxygenase expression testing}

243 To test expression of the $N$-oxygenase ladder, $5 \mathrm{~mL}$ cultures of NB17-NB37 were inoculated

244 in $5 \mathrm{~mL}$ cultures of LBL containing $50 \mu \mathrm{g} / \mathrm{mL}$ kanamycin and then grown at $37^{\circ} \mathrm{C}$ until mid- 
245 exponential phase $(\mathrm{OD}=0.5-0.8)$. At this time, cultures were induced via addition of $0.2 \mathrm{nM}$ aTc

246 and then grown at $30^{\circ} \mathrm{C}$ for $5 \mathrm{~h}$ before growing at $20^{\circ} \mathrm{C}$ for an additional $18 \mathrm{~h}$. After this time, 1

$247 \mathrm{~mL}$ of cells was mixed with $0.05 \mathrm{~mL}$ of glass beads and then vortexed using a Vortex Genie 2 for

24815 minutes. After this time, the lysate was centrifuged at $18,213 \mathrm{~g}$ at $4{ }^{\circ} \mathrm{C}$ for 30 minutes. Lysate

249 was denatured as described in Section 2.5 and then subsequently run on an SDS-PAGE gel with

250 an Invitrogen BenchMark ${ }^{\mathrm{TM}}$ His-tagged Protein Standard ladder and then analyzed via western

251 blot with an HRP-conjugated 6*His His-Tag Mouse McAB primary antibody. The blot was

252 visualized using an Amersham ECL Prime chemiluminescent detection reagent.

$253 \quad$ 2.6.In vitro $N$-oxygenase activity assay

254 Reactions were performed in $1 \mathrm{~mL}$ volumes consisting of $25 \mathrm{mM}$ HEPES pH 7.0, $25 \mathrm{mM}$

$255 \mathrm{NaCl}$, and $1.5 \% \mathrm{H}_{2} \mathrm{O}_{2}$ with $1 \mathrm{mM}$ pA-Phe or pA-Pyr. The reaction mixture was incubated for $3 \mathrm{~h}$

256 at $25{ }^{\circ} \mathrm{C}$ with $10 \mu \mathrm{M}$ purified $N$-oxygenase, following which protein was removed by filtering

257 through a $10 \mathrm{~K}$ Amicon centrifugal filter unit. The eluent was then analyzed by HPLC. pN-Phe

258 production was further confirmed via UPLC-MS.

259 2.7.HPLC Analysis

260 Metabolites of interest were quantified via high-performance liquid chromatography (HPLC)

261 using an Agilent 1260 Infinity model equipped with a Zorbax Eclipse Plus-C18 column. To

262 quantify amine containing metabolites, an initial mobile phase of solvent $\mathrm{A} / \mathrm{B}=100 / 0$ was used

263 (solvent A, $20 \mathrm{mM}$ potassium phosphate, $\mathrm{pH}$ 7.0; solvent B, acetonitrile/water at 50/50) and

264 maintained for $7 \mathrm{~min}$. A gradient elution was performed (A/B) with: gradient from 100/0 to 50/50

265 for 7-17 min, gradient from 50/50 to 100/00 for 17-18 min, equilibration at 100/0 for 18-22 min.

266 A flow rate of $0.5 \mathrm{~mL} \mathrm{~min}^{-1}$ was maintained and absorption was monitored at 210 and $320 \mathrm{~nm}$. To

267 quantify nitro-group containing metabolites, we used an initial mobile phase of solvent $\mathrm{A} / \mathrm{B}=$ 
100/0 (solvent A, water, $0.1 \%$ trifluoroacetic acid; solvent B, acetonitrile, $0.1 \%$ trifluoroacetic acid) and maintained for 5 min. A gradient elution (A/B) was then performed with: gradient from $100 / 0$ to $95 / 5$ over 5-7 min, gradient from $95 / 5$ to $90 / 10$ over $7-10 \mathrm{~min}$, gradient from $90 / 10$ to $80 / 20$ over $10-16$ min, gradient from $80 / 20$ to $70 / 30$ over $16-19$ min, gradient from $70 / 30$ to $0 / 100$ over 19-21 min, 0/100 over 21-23 min, gradient from 0/100 to 100/0 over 23-24 min, and equilibration at 100/0 over $24-25$ min. The nitro product quantifying method used flow rate of 0.5

$274 \mathrm{~mL} \mathrm{~min}^{-1}$ and monitored absorption at 280 and $320 \mathrm{~nm}$.

\subsection{Mass Spectrometry}

Mass spectrometry (MS) measurements for small molecule metabolites were submitted to a Waters Acquity UPLC H-Class coupled to a single quadrupole mass detector 2 (SQD2) with an 278 electrospray ionization source. Metabolite compounds were analyzed using a Waters Cortecs 279 UPLC C18 column with an initial mobile phase of solvent A/B = 95/5 (solvent A, water, $0.1 \%$

280 formic acid; solvent B, acetonitrile, $0.1 \%$ formic acid) with a gradient elution from (A/B) 95/5 to $2815 / 95$ over $5 \mathrm{~min}$. Flow rate was maintained at $0.5 \mathrm{~mL} \mathrm{~min}^{-1}$. For samples collected from E. coli 282 growth cultures, an initial submission to an Agilent 1100 series HPLC system with a Zorbax 283 Eclipse Plus C18 column was used to collect $\mathrm{pN}$-Phe elution peaks for enhanced MS resolution. A $100 \mathrm{uL}$ injection was made with an initial mobile phase of solvent $\mathrm{A} / \mathrm{B}=95 / 5$ (solvent $\mathrm{A}$, water, $2850.1 \%$ trifluoroacetic acid; solvent B, acetonitrile, $0.1 \%$ trifluoroacetic acid) and maintained for 1 min. A gradient elution was then performed (A/B) with: gradient from 95/5 to 50/50 over 1-24 287 min, gradient from $50 / 50$ to $95 / 5$ over $24-25$ min, equilibration at $95 / 5$ for $25-27$ min. Flow rate was $1 \mathrm{~mL} / \mathrm{min}$ and metabolites were tracked at $270 \mathrm{~nm}$. pN-Phe elution was identified at $7.20 \mathrm{~min}$ using a chemical standard and this peak was collected for submission to UPLC-MS. 


\section{Results}

\subsection{Pathway Metabolite Stability and Toxicity}

To initially determine whether the nitroaromatic compounds and their amine precursors in our

Here, we supplemented $1 \mathrm{mM}$ of our envisioned pathway metabolites (pA-Phe, pA-Pyr, pN-Phe,

5\% DMSO to aid metabolite solubility. We grew cultures and monitored cell density, observing

Pyr, which increased doubling time from $27.4 \pm 0.1 \mathrm{~min}$ to $54.8 \pm 0.6 \mathrm{~min}$ (Fig. 2A). Fortunately, neither the aromatic amines nor pN-Phe exhibited notable toxicity.

We then performed stability testing in a similar manner, with candidate metabolites added at instability would be convenient for our pathway design as the final pathway step would be to $\mathrm{pN}-\mathrm{Ph}$. 
A

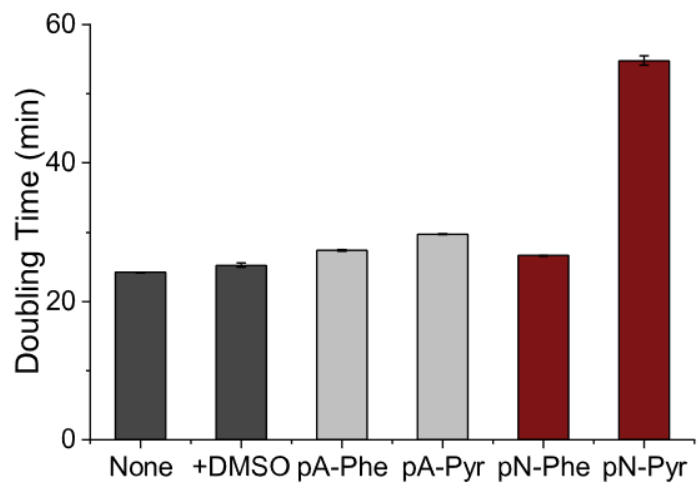

B

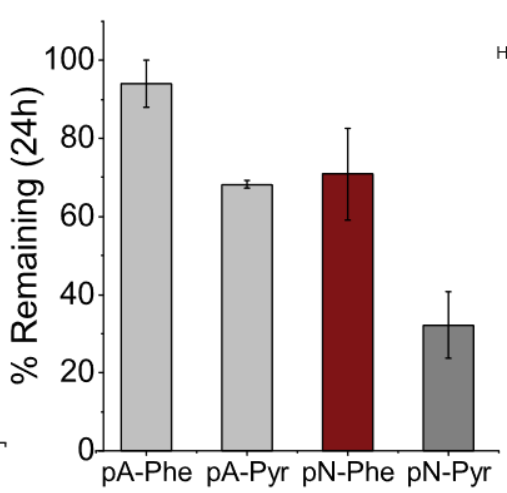

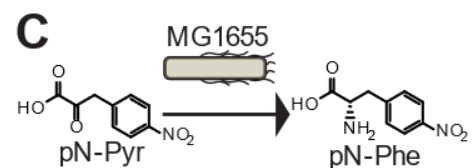

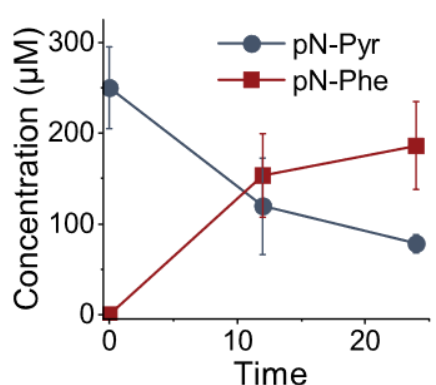

Figure 2. Assessment of pathway metabolite stability and toxicity. (A) Toxicity testing of pathway metabolites pA-Phe, pA-Pyr, pN-Phe, and pN-Pyr in E. coli MG1655(DE3) in with no additives (None), 5\% DMSO for solubility (+DMSO), or $1 \mathrm{mM}$ addition of associated metabolite with 5\% DMSO measured via E. coli doubling time. (B) Stability testing of pathway metabolites measured via the percent of the initial metabolite supplementation $(0.5 \mathrm{mM})$ remaining after $24 \mathrm{~h}$ of fermentative growth in at $37^{\circ} \mathrm{C}$. (C) Tracking of pN-Pyr conversion to pN-Phe in cultures supplemented with $0.25 \mathrm{mM}$ pN-Pyr over a $24 \mathrm{~h}$ fermentation in E. coli MG1655(DE3). All cultures were grown in LBL media. Sample size is $n=3$ using biological replicates. Data shown are mean \pm standard deviation. 


\subsection{Initial $\mathrm{N}$-oxygenase Characterization}

326

327

328

329

330

331

332

333

Given that E. coli synthesis of pA-Phe and pA-Pyr has previously been established, we sought to identify an $N$-oxygenase that can fully oxidize pA-Phe or pA-Pyr. There are four distinct types of $N$-oxygenase that have been characterized to date (Nóbile et al., 2021): (i) Flavin-dependent monooxygenases, (ii) Heme-oxygenase-like diiron oxygenases, (iii) Rieske-type $N$-oxygenases, and (iv) Non-heme diiron monooxygenases. Investigation into flavin-dependent monooxygenases has shown that they commonly act on amine-containing sugars (Huynh et al., 2020; Thoden et al., 2013). Additionally, studies on the substrate scope of heme-oxygenase-like diiron oxygenases have been fairly limited (imidazole and $\mathrm{N}^{\omega}$-methyl-1-arginine) (Hedges and Ryan, 2019; Ng et al., 2019). While the Rieske-type $N$-oxygenase PrnD has previously demonstrated activity on pAPhe, reconstitution of Rieske $N$-oxygenases in vivo has been challenging in $E$. coli due to limits in iron-sulfur cluster incorporation into enzymatic cores (Tiwari et al., 2011). Previous studies have demonstrated the activity of the characterized non-heme diiron monooxygenase-type $N$ oxygenases AurF (Winkler and Hertweck, 2005) and CmlI (Lu et al., 2012) in vivo, but neither have been shown to effectively convert pA-Phe to $\mathrm{pN}$-Phe (Chanco et al., 2014). More recent work identified putative diiron monooxygenase $\mathrm{ObaC}$ in the synthesis of obafluorin. In that work, the activity of $\mathrm{ObaC}$ on pA-Pyr was indirectly demonstrated via a colorimetric assay wherein NADH oxidation led to color shift in the presence of phenazine methosulfate, iron(II) sulfate and ObaC (Schaffer et al., 2017). Additional combination of ObaC with $\mathrm{ObaH}$ and $\mathrm{ObaG}$ identified a nitrocontaining final product via mass spectrometry, but confirmation of full oxidation of pA-Pyr to pN-Pyr was not measured via mass spectrometry. To confirm ObaC activity on pA-Pyr and test activity on pA-Phe, we prepared three ObaC fusions $\left(\mathrm{ObaC}-\mathrm{C}_{\text {term }} \mathrm{His}_{6 \mathrm{x}}, \mathrm{N}_{\text {term }} \mathrm{His}_{6 \mathrm{x}}-\mathrm{ObaC}\right.$, and $\mathrm{N}_{\text {term }} \beta$ gal-ObaC-C $\mathrm{C}_{\text {termHis6x }}$ [ßgal-ObaC]) and performed Ni-NTA chromatography. We isolated 
348 proteins of correct size for NtermHis6x-ObaC and $\beta g a l-O b a C$, but were unable to isolate ObaC-

349 CtermHis6x. We then performed in vitro characterization on $\mathrm{N}_{\text {term } H i s 6 \mathrm{x}-O b a C}$ and $\beta$ gal-ObaC

350 using $\mathrm{H}_{2} \mathrm{O}_{2}$ as a reductant to recycle the enzyme's diiron core (Chanco et al., 2014). Using $10 \mu \mathrm{M}$

351 purified enzymes, we ran reactions containing $1.5 \% \mathrm{H}_{2} \mathrm{O}_{2}$ over $3 \mathrm{~h}$ with $1 \mathrm{mM}$ of either pA-Pyr or

352 pA-Phe. Our initial results indicated that $\mathrm{N}_{\text {term }}$ His $6 \mathrm{x}-\mathrm{ObaC}$ was unable to achieve sufficient activity

353 on pA-Pyr or pA-Phe for measurement on HPLC. However, reactions run with pA-Pyr and pA-

354 Phe using $\beta$ gal-ObaC obtained $127 \pm 6 \mu \mathrm{M}$ pN-Pyr and $105 \pm 15 \mu \mathrm{M}$ pN-Phe, respectively (Fig.

355 3A). Using UPLC-MS analysis, the pA-Phe supplemented experiment was confirmed to produce 356 pN-Phe.

357 To validate $\mathrm{ObaC}$ activity in vivo, we performed a supplementation experiment with an 358 MG1655 strain harboring a pZE plasmid vector bearing the $o b a C$ gene. Here, we grew cultures to 359 mid-exponential phase, and then supplemented cultures with either pA-Pyr or pA-Phe and induced 360 ObaC expression. These experiments resulted in extracellular production of $31 \pm 1 \mu \mathrm{M}$ pN-Phe 361 with pA-Pyr supplementation or $240 \pm 20 \mu \mathrm{M}$ pN-Phe with pA-Phe supplementation after $24 \mathrm{~h}$ 362 (Fig. 3B), demonstrating that ObaC could act on either pathway intermediate. Our low titers 363 suggest that $\mathrm{pA}-\mathrm{Pyr}$ or $\mathrm{pA}-\mathrm{Phe}$ may be transport-limited upon supplementation and that $\mathrm{pA}-\mathrm{Phe}$ 364 may enter cells more easily than pA-Pyr. 
A<smiles>[R]C(Cc1ccc(N)cc1)C(=O)O</smiles>

$\mathrm{R}:=\mathrm{O}(\mathrm{pA}-\mathrm{Pyr})$ or $\mathrm{NH}_{2}$ (pA-Phe)
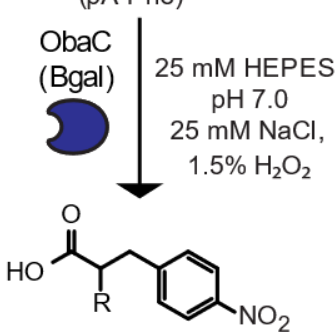

$\mathrm{R}:=\mathrm{O}(\mathrm{pA}-\mathrm{Nyr})$ or $\mathrm{NH}_{2}$

B (pN-Phe)<smiles>[R]C(Cc1ccc(N)cc1)C(=O)O</smiles><smiles>[R]O[18O][18O][18O]</smiles><smiles>[R]OC(=O)O[R16]([R])([H])[H]</smiles><smiles>N[C@@H](Cc1ccc([N+](=O)[O-])cc1)C(=O)O</smiles>

pN-Phe
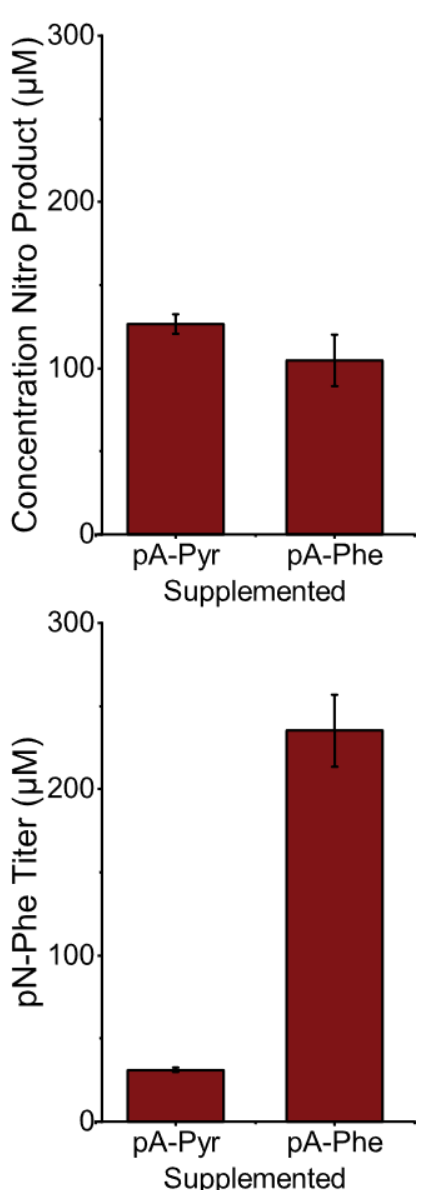

366 Figure 3. Evaluation of the activity of the $N$-oxygenase $\mathrm{ObaC}$ on the aromatic amines pA-Pyr and 367 pA-Phe. (A) In vitro investigation of ObaC activity on pA-Phe and pA-Pyr. (B) In vivo 368 supplementation testing of MG1655(DE3) expressing $\beta$ gal-ObaC in the conversion of $2 \mathrm{mM}$ supplemented pA-Pyr or pA-Phe to pN-Phe end product after $24 \mathrm{~h}$ of fermentative growth. Sample size is $n=3$ using biological replicates. Data shown are mean \pm standard deviation. 


\subsection{Implementation of a de novo $\mathrm{pN}$-Phe synthesis pathway}

372

373

374

375

376

377

378

379

380

381

382

383

384

385

386

387

388

389

390

391

392

393

Given demonstrated activity of $\mathrm{ObaC}$ on desired aromatic amines and native conversion of pN-Pyr to pN-Phe, we then sought to confirm synthesis of pA-Phe using the PapABC enzymes. We chose to utilize the papABC genes from $S$. venezuelae rather than homologues in $P$. fluorescens given results from a previous study that suggested that the $S$. venezuelae enzymes result in greater pA-Phe titer (Chen et al., 2018). To start, we obtained the papABC operon from the Mehl Lab in the pLASC-lppPW plasmid and then moved these into an IPTG inducible pCola plasmid under the control of an IPTG-inducible T7-promoter system. Initial shake flask testing of this construct in $E$. coli MG1655(DE3) using M9-glucose minimal media resulted in low 24h post-induction pA-Phe titers of $<10 \mu \mathrm{M}$ (Fig. 4A, NB06). Expression of the feedback-resistant DAHP aldolase AroG*, which is known to enhance shikimate pathway flux (Kunjapur et al., 2014), was tested using an IPTG-inducible pACYC plasmid under the control T7-promoter system with pCola-papABC (NB07). This strain resulted in significant $24 \mathrm{~h}$ post-induction pA-Phe titer of $624 \pm 79 \mu \mathrm{M}$.

We then incorporated the $\mathrm{N}$-oxygenase $\mathrm{ObaC}$ into the pathway in a format using two plasmids: (i) a pACYC vector expressing $\mathrm{AroG}^{*}$ and $\mathrm{ObaC}$ in an operon and (ii) the pCola vector expressing the PapABC operon (NB08). Here we first obtained de novo biosynthesis of pN-Phe, with a titer of $83 \pm 13 \mu \mathrm{M}$ pN-Phe and $46 \pm 5 \mu \mathrm{M}$ pA-Phe (Fig. 4A). We confirmed that the product mass of the peak of interest corresponded to pN-Phe by UPLC-MS analysis of a second NB08 fermentation in minimal media supplemented with glucose. Then, we cloned a single pCola vector expressing AroG* and $\mathrm{ObaC}$ in an IPTG-inducible operon and PapABC on an additional IPTG-inducible operon and expressed this in MG1655 (DE3) (NB09) (Fig. 4B). This strain demonstrated superior $\mathrm{pN}$-Phe synthesis achieving a higher $24 \mathrm{~h}$ post-induction titer of $\mathrm{pN}$-Phe of $149 \pm 5 \mu \mathrm{M}$ in addition to a higher titer of $\mathrm{pA}-\mathrm{Phe}$ of $153 \pm 22 \mu \mathrm{M}$. 

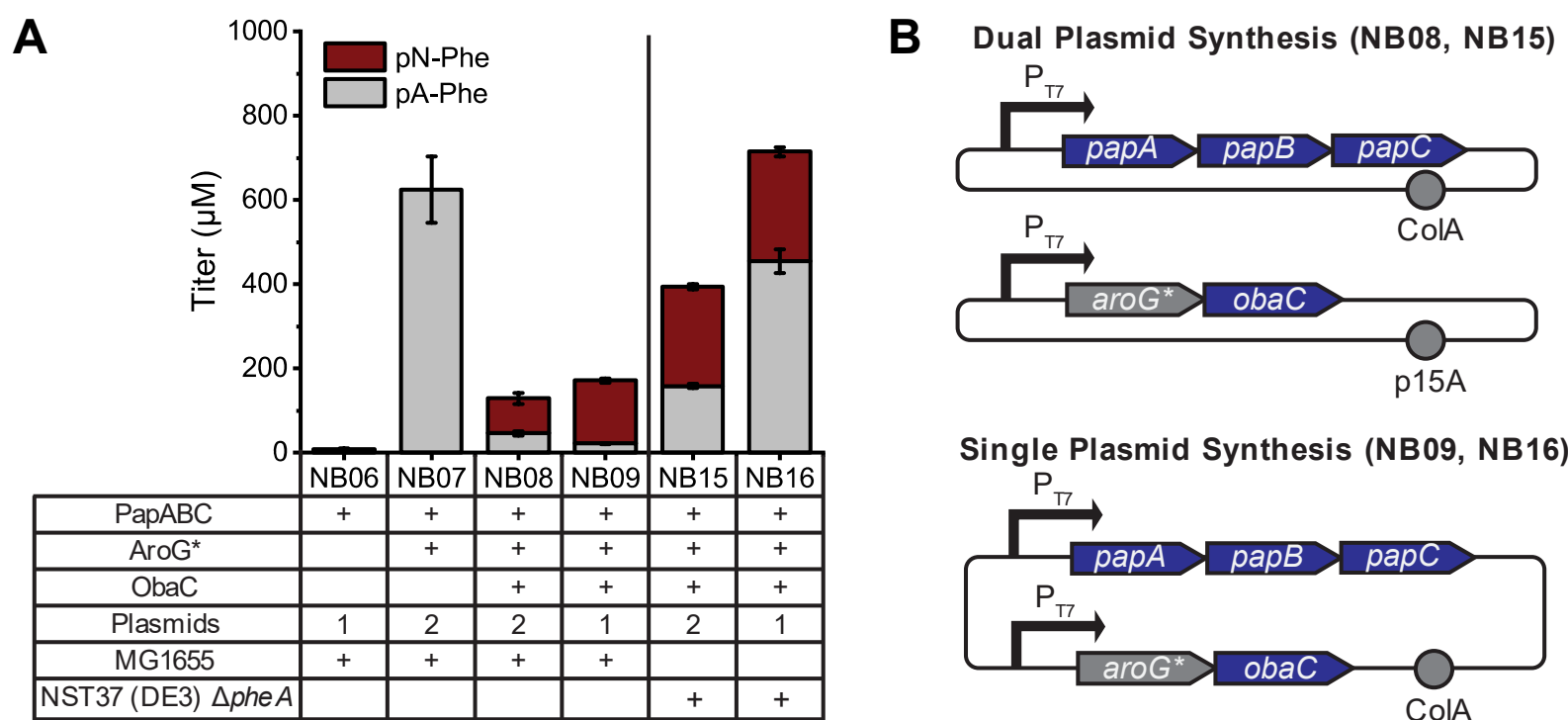

Single Plasmid Synthesis (NB09, NB16)
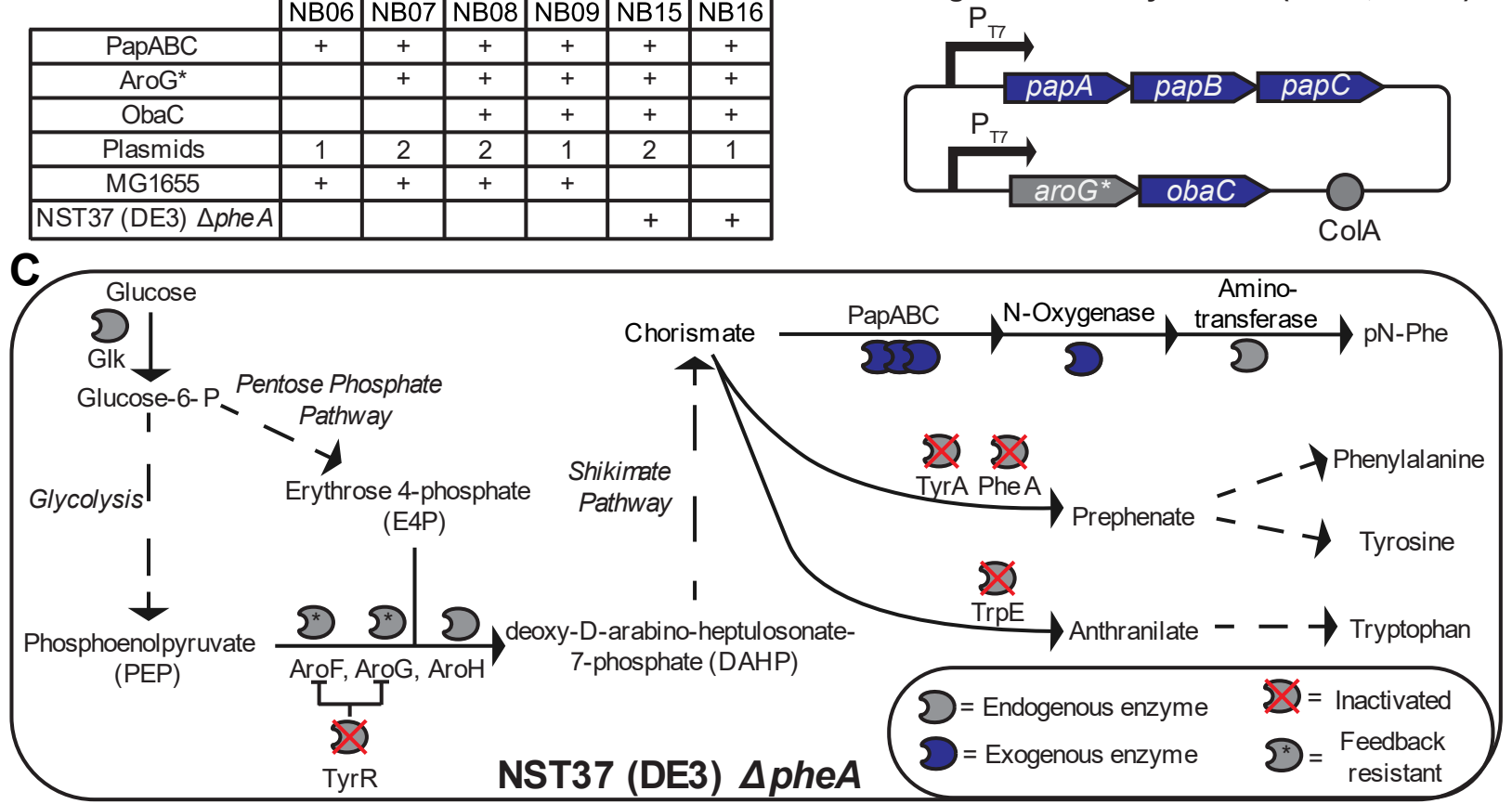

Figure 4. De novo synthesis of $\mathrm{pN}$-Phe in E. coli. (A) Titer of $\mathrm{pA}-\mathrm{Phe}$ and $\mathrm{pN}-\mathrm{Phe}$ in different strains expressing pathway enzymes following $24 \mathrm{~h}$ of fermentation in M9-glucose (1.5\%) minimal media Sample size is $n=3$ using biological replicates. Data shown are mean \pm standard deviation. (B) Depiction of the different arrangement of pathway plasmids for the synthesis of pN-Phe. (C) Depiction of the E. coli NST37(DE3) $\triangle$ PheA strain engineered for enhanced pN-Phe titer through chorismate. Inactivated enzymes are shown with a red cross and feedback-resistant versions of enzymes are shown with a star (*). GIK, glucokinase; AroG, phenylalanine-sensitive Phospho-2dehydro-3-deoxyheptonate (DAHP) synthase; AroF, tyrosine-sensitive DAHP synthase; AroH, tryptophan-sensitive DAHP synthase; TyrA and PheA, TyrA and PheA subunits of the chorismate mutase; TrpE, anthranilate synthase component I; TyrR, transcriptional regulatory protein. 
To further enhance titer, we purchased a commercially available phenylalanine overproducer strain and performed several modifications to improve its suitability for $\mathrm{pN}$-Phe biosynthesis. We inactivated the expression of the initial enzyme which converts chorismate flux to phenylalanine,

408 PheA (Rodriguez et al., 2014). We additionally performed genomic integration of the T7409 polymerase and $l a c I$ and $l a c Z$ genes for use with our plasmid setup, creating the NST37 (DE3) $410 \Delta p h e A$ strain. This strain is unable to express functional proteins for the synthesis of all three 411 standard aromatic amino acids (TyrA, TrpE, PheA) and a shikimate pathway regulator (TyrR). 412 Furthermore, the strain expresses feedback inhibition enzyme variants (AroF* and AroG*) to 413 enhance shikimate pathway flux, which should result in greater accumulation of chorismate (Fig.

414 4C). Using this modified strain, we transformed both the two-plasmid system as previously 415 described (NB15) and the single plasmid system (NB16) and performed shake-flask fermentation over $24 \mathrm{~h}$ in M9-glucose minimal media. Here, we achieved our highest measured titer of $260 \pm$

$41711 \mu \mathrm{M}$ using the single plasmid system. Despite promising $\mathrm{pN}$-Phe titer, in the case of the single 418 plasmid system, the unconverted pA-Phe titer remained quite high at $454 \pm 28 \mu \mathrm{M}$.

\subsection{Broad $N$-oxygenase screening and pathway implementation}

421 proteins have supplemented $1 \mathrm{mM}$ of the nsAA into culture to achieve incorporation (Grünewald 422 et al., 2008; Tsao et al., 2006), but lower concentrations of nsAA were not tested. While the 423 obtained titer of approximately $250 \mu \mathrm{M}$ may be sufficient for incorporation, high accumulation of 424 pA-Phe could result in off-target nsAA incorporation if a strain were to perform biosynthesis and 425 site-specific nsAA incorporation. Additionally, poor selectivity of pN-Phe biosynthesis would 426 complicate downstream isolation of the small molecule product if that option were to be pursued. 427 Thus, to improve product titer and make $\mathrm{pN}$-Phe the dominant nsAA product, we sought to identify 
428 an $\mathrm{N}$-oxygenase enzyme with improved activity on pA-Phe. Previously characterized non-heme

429 diiron monooxygenases have demonstrated activity on diverse aromatic amines and compatibility

430 with expression in E. coli, but $\mathrm{ObaC}$ is the only diiron monooxygenase thus far to exhibit full

431 oxidation of pA-Phe to pN-Phe. To assess the broader space of $N$-oxygenase enzymes, we used

432 NCBI BLAST to obtain the 1000 most closely related sequences as measured by BLASTP

433 alignment score from four characterized diiron monooxygenase-type $N$-oxygenases with activity

434 on aromatic amines: AurF, CmlI, AzoC (Guo et al., 2019), and ObaC. After deleting duplicate

435 sequences, we obtained 2134 unique sequences which we then submitted to the Enzyme Function

436 Initiative-Enzyme Similarity Tool (EFI-EST) (Gerlt et al., 2015) to generate a sequence similarity

437 network (SSN). Sequences exhibiting greater than 95\% similarity were grouped into single nodes,

438 resulting in 775 unique nodes and a minimum alignment score of 100 was selected for node edges.

439 We visualized the SSN using Cytoscape (Fig. 5). 


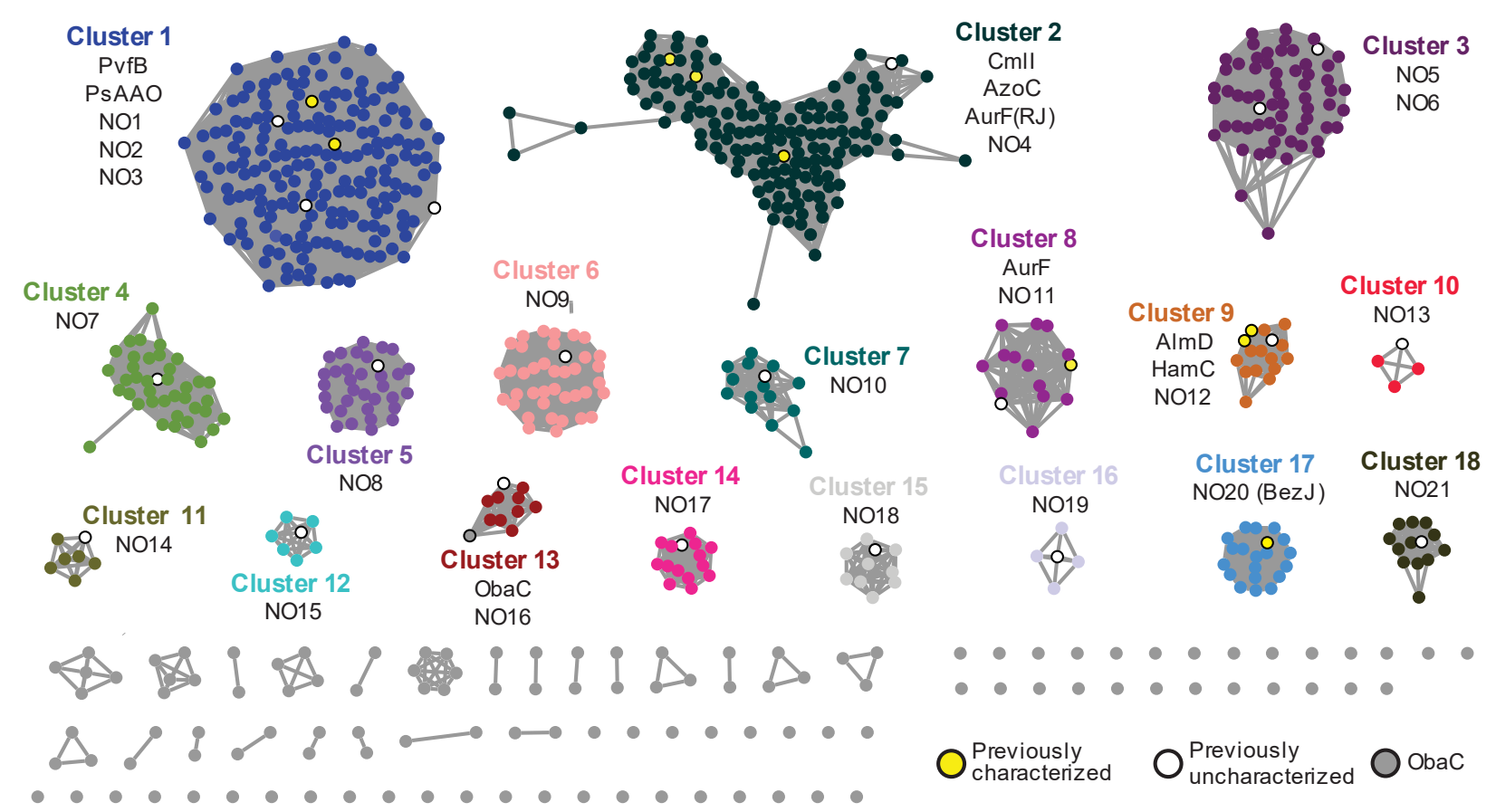

441

442

443

444

445

446 (Kretsch et al., 2018), PsAAO (Platter et al., 2011), CmlI (Knoot et al., 2016; Lu et al., 2012),

447 AzoC (Guo et al., 2019), AurF(RJ) (Indest et al., 2015), AurF (Seong Choi et al., 2008), AlmD

448 (Cortina et al., 2011), HamC (Jenul et al., 2018), and BezJ (Tsutsumi et al., 2018)), putative $N$ -

Figure 5. Sequence similarity network generated using 2134 unique putative diiron monooxygenase sequences determined from NCBI BLAST of the $N$-oxygenases AurF, CmlI, AzoC, and $\mathrm{ObaC}$ represented as 775 unique nodes. Edges are drawn between nodes with minimum alignment score of 100. Previously characterized $N$-oxygenases are highlighted in yellow (PvfB oxygenases cloned and tested in this study are highlighted in white, and $\mathrm{ObaC}$ is shown in gray with black outline. 
To test a diverse set of $N$-oxygenase enzymes, we selected twenty-one distinct sequences from

45218 different clusters to obtain diversity in the diiron monooxygenase sequence space from those

453 currently characterized and ordered gene fragments to clone these into a pZE expression vector

454 with a C-terminal hexahistidine tag. All but one of these $\mathrm{N}$-oxygenases (NO20, also known as

455 BezJ) (Tsutsumi et al., 2018)) were previously uncharacterized. We initially tested the expression

456 of these proteins in E. coli by transforming them into MG1655(DE3) for subsequent overnight

457 expression. Analysis of the lysate using anti-His western blotting confirmed the soluble synthesis

458 of all the $N$-oxygenases except NO6, NO13, NO18, and NO20. We then tested the $N$-oxygenases

459 in MG1655(DE3) by growing them in LBL media to mid-exponential phase, at which time, we

460 supplemented cultures with $1 \mathrm{mM}$ pA-Phe and induced $N$-oxygenase expression. Fermentation

461 with metabolically active cells for $24 \mathrm{~h}$ at $30^{\circ} \mathrm{C}$ revealed that only one of the additional $N$ -

462 oxygenases resulted in $\mathrm{pN}$-Phe production apart from $\mathrm{ObaC}$. This $N$-oxygenase was NO16 from

463 Pseudomonas sp. EMN2, which exhibited greater than twice the pN-Phe production in the initial

464 screen (Table 1). Previous reports of para-aminobenzoic acid testing have noted activity in

465 enzymes across different clusters (PsAAO (Platter et al., 2011), AurF (Seong Choi et al., 2008),

466 and HamC (Jenul et al., 2018)), which we did not observe for pA-Phe. Following the initial in vivo

467 screen, we purified NO16 with a C-terminal hexahistidine tag and performed a $3 \mathrm{~h}$ in vitro reaction

468 containing $1.5 \% \mathrm{H}_{2} \mathrm{O}_{2}$ with $1 \mathrm{mM}$ of either pA-Pyr or pA-Phe. In comparison to ObaC, we

469 obtained similar titer of $\mathrm{pN}-\mathrm{Pyr}$ in this case $(120 \pm 3 \mu \mathrm{M}$ final concentration $\mathrm{pN}-\mathrm{Pyr})$ and a two-

470 fold increase in the titer of $\mathrm{pN}$-Phe $(196 \pm 23 \mu \mathrm{M}$ final concentration of $\mathrm{pN}-\mathrm{Phe})$ when NO16 is

471 used as catalyst (Fig. 6A). 
472 Table 1. In vivo screening of $N$-oxygenase activity on pA-Phe via supplementation testing of 1

$473 \mathrm{mM}$ pA-Phe in cultures expressing $N$-oxygenases with $\mathrm{C}$-terminal hexahistidine tags via

474 measurement of $\mathrm{pN}$-Phe titer after $24 \mathrm{~h}$ fermentation at $37^{\circ} \mathrm{C}$. ObaC was tested without a

475 hexahistidine tag.

476

477

\begin{tabular}{|l|c|}
\hline $\boldsymbol{N}$-oxygenase & $\begin{array}{c}\mathbf{p N}-P h e \text { Titer } \\
(\boldsymbol{\mu M})\end{array}$ \\
\hline ObaC & $45 \pm 19$ \\
\hline $\begin{array}{l}\text { NO6, NO13, NO18, } \\
\text { NO20 }\end{array}$ & $\begin{array}{c}\text { No protein } \\
\text { detected }\end{array}$ \\
\hline $\begin{array}{l}\text { NO1-5, NO7-12, NO14, } \\
\text { NO15, NO17, NO19, } \\
\text { NO21 }\end{array}$ & $\begin{array}{c}\text { No product } \\
\text { detected }\end{array}$ \\
\hline NO16 & $105 \pm 39$ \\
\hline
\end{tabular}


A<smiles>[R]C(Cc1ccc(N)cc1)C(=O)O</smiles>

$\mathrm{R}:=\mathrm{O}(\mathrm{pA}-\mathrm{Pyr})$ or $\mathrm{NH}_{2}$ (pA-Phe)

25 mM HEPES, NO16

$\mathrm{pH} 7.0$

$25 \mathrm{mM} \mathrm{NaCl}$,

$1.5 \% \mathrm{H}_{2} \mathrm{O}_{2}$

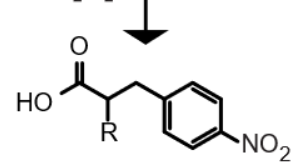

$\mathrm{R}:=\mathrm{O}(\mathrm{pA}-\mathrm{Nyr})$ or $\mathrm{NH}_{2}$ (pN-Phe)

B

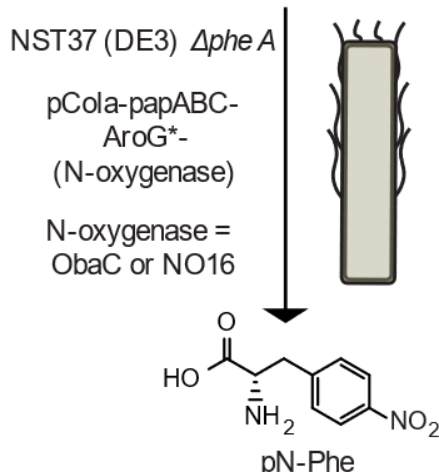

pN-Phe

MOPS-EZ Rich-Glucose

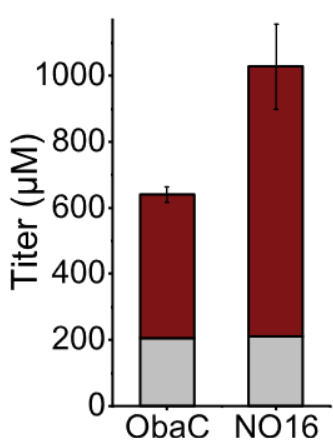

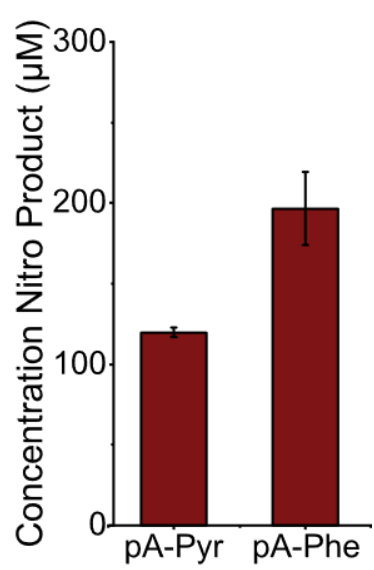

Supplemented

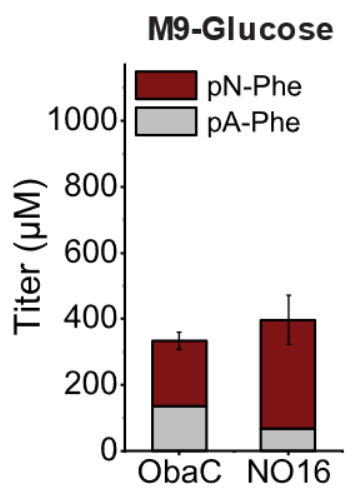

Figure 6. Evaluation of the $N$-oxygenase NO16 in the synthesis of pN-Phe. (A) In vitro

481 investigation of NO16 activity on pA-Phe and pA-Pyr. (B) Media comparison of the pN-Phe and

482 pA-Phe titers using the NST(DE3) $\Delta p h e A$ strain of $E$. coli transformed with either pCola-papABC-

483 AroG*-ObaC (strain NB16) or pCola-papABC-AroG*-NO16 (strain NB39) in either M9-glucose,

484 MOPS-EZ rich-glucose, or LBL-glucose media. 
Then, we sought to compare the performance of $\mathrm{NO} 16$ and $\mathrm{ObaC}$ within our de novo NO16 in place of ObaC in the operon with $\operatorname{aroG}^{*}$. We then evaluated the $24 \mathrm{~h}$ titer in M9-glucose minimal media of the pCola-papABC-AroG*-NO16 construct in the NST37 (DE3) $\Delta p h e A$ strain compared to the strain expressing ObaC $(197 \pm 25 \mu \mathrm{M})$ (Fig. 6B).

With this new $N$-oxygenase in hand, we finally evaluated the $\mathrm{pN}$-Phe titer observed at $24 \mathrm{~h}$ defined media, we saw the highest pN-Phe titers of $820 \pm 130 \mu \mathrm{M}$ when using NO16 in the pCola vector compared to $435 \pm 23 \mu \mathrm{M}$ when using ObaC (Fig. 6B). In richer LBL-glucose media, we media.

\section{Conclusion}

This work is the first to demonstrate de novo biosynthesis of a non-naturally occurring nsAA. renewable feedstocks is valuable, and further studies into nitroaromatic biosynthesis using $N$ - 
508 Utilization of this pathway with methods of genetic code expansion could enable the use of $\mathrm{pN}$ -

509 Phe within proteins for in situ applications and further broaden its potential use case.

510

511 5. References

512 Arbely, E., Torres-Kolbus, J., Deiters, A., Chin, J.W., 2012. Photocontrol of tyrosine

513 phosphorylation in mammalian cells via genetic encoding of photocaged tyrosine. J. Am.

514 Chem. Soc. 134, 11912-11915. https://doi.org/10.1021/ja3046958

515 Badgujar, D.M., Talawar, M.B., Mahulikar, P.P., 2016. Review on greener and safer synthesis of 516 nitro compounds. Propellants, Explos. Pyrotech. 41, 24-34. https://doi.org/10.1002/prep.201500090

Barry, S.M., Kers, J.A., Johnson, E.G., Song, L., Aston, P.R., Patel, B., Krasnoff, S.B., Crane, B.R., Gibson, D.M., Loria, R., Challis, G.L., 2012. Cytochrome P450-catalyzed Ltryptophan nitration in thaxtomin phytotoxin biosynthesis. Nat. Chem. Biol. 8, 814-816. https://doi.org/10.1038/nchembio.1048

Booth, G., 2000. Nitro Compounds, Aromatic, in: Ullmann's Encyclopedia of Industrial

Bryantsev, V.S., Hay, B.P., 2005. Influence of substituents on the strength of aryl C-H $\cdots$ anion https://doi.org/10.1002/14356007.a17_411

527 Caranto, J.D., 2019. The emergence of nitric oxide in the biosynthesis of bacterial natural products. Curr. Opin. Chem. Biol. 49, 130-138. https://doi.org/10.1016/j.cbpa.2018.11.007 
Chanco, E., Choi, Y.S., Sun, N., Vu, M., Zhao, H., 2014. Characterization of the N-oxygenase AurF from Streptomyces thioletus. Bioorganic Med. Chem. 22, 5569-5577. https://doi.org/10.1016/j.bmc.2014.06.002

Chen, P.R., Groff, D., Guo, J., Ou, W., Cellitti, S., Geierstanger, B.H., Schultz, P.G., 2009. A facile system for encoding unnatural amino acids in mammalian cells. Angew. Chemie Int. Ed. 48, 4052-4055. https://doi.org/10.1002/anie.200900683

Chen, Y., Loredo, A., Gordon, A., Tang, J., Yu, C., Ordonez, J., Xiao, H., 2018. A noncanonical amino acid-based relay system for site-specific protein labeling. Chem. Commun. 54, 71877190. https://doi.org/10.1039/C8CC03819H

Chen, Y., Tang, J., Wang, L., Tian, Z., Cardenas, A., Fang, X., Chatterjee, A., Xiao, H., 2020. Creation of bacterial cells with 5-hydroxytryptophan as a 21st amino acid building block. Chem 6, 2717-2727. https://doi.org/10.1016/j.chempr.2020.07.013

Cortina, N.S., Revermann, O., Krug, D., Müller, R., 2011. Identification and characterization of the althiomycin biosynthetic gene cluster in Myxococcus xanthus DK897. ChemBioChem 12, 1411-1416. https://doi.org/10.1002/CBIC.201100154

Cui, L., Shearwin, K.E., 2017. Clonetegration using OSIP plasmids: One-step DNA assembly and site-specific genomic integration in bacteria, in: Methods in Molecular Biology. Humana Press, New York, NY, pp. 139-155. https://doi.org/10.1007/978-1-4939-63430_11

Deiters, A., Groff, D., Ryu, Y., Xie, J., Schultz, P.G., 2006. A genetically encoded photocaged tyrosine. Angew. Chemie Int. Ed. 45, 2728-2731. https://doi.org/10.1002/anie.200600264 
Dickey, R.M., Forti, A.M., Kunjapur, A.M., 2021. Advances in engineering microbial biosynthesis of aromatic compounds and related compounds. Bioresour. Bioprocess. 8, 91. https://doi.org/10.1186/S40643-021-00434-X

Dodani, S.C., Kiss, G., Cahn, J.K.B., Su, Y., Pande, V.S., Arnold, F.H., 2016. Discovery of a regioselectivity switch in nitrating $\mathrm{P} 450$ s guided by molecular dynamics simulations and Markov models. Nat. Chem. 201685 8, 419-425. https://doi.org/10.1038/nchem.2474

Gauba, V., Grunewald, J., Gorney, V., Deaton, L.M., Kang, M., Bursulaya, B., Ou, W., Lerner, R.A., Schmedt, C., Geierstanger, B.H., Schultz, P.G., Ramirez-Montagut, T., 2011. Loss of CD4 T-cell-dependent tolerance to proteins with modified amino acids. Proc. Natl. Acad. Sci. 108, 12821-12826. https://doi.org/10.1073/pnas.1110042108

Gautier, A., Nguyen, D.P., Lusic, H., An, W., Deiters, A., Chin, J.W., 2010. Genetically encoded photocontrol of protein localization in mammalian cells. J. Am. Chem. Soc. 132, 40864088. https://doi.org/10.1021/ja910688s

Gerlt, J.A., Bouvier, J.T., Davidson, D.B., Imker, H.J., Sadkhin, B., Slater, D.R., Whalen, K.L., 2015. Enzyme function initiative-enzyme similarity tool (EFI-EST): A web tool for generating protein sequence similarity networks. Biochim. Biophys. Acta - Proteins Proteomics 1854, 1019-1037. https://doi.org/10.1016/j.bbapap.2015.04.015

Grünewald, J., Hunt, G.S., Dong, L., Niessen, F., Wen, B.G., Tsao, M.L., Perera, R., Kang, M., Laffitte, B.A., Azarian, S., Ruf, W., Nasoff, M., Lerner, R.A., Schultz, P.G., Smider, V. V., 2009. Mechanistic studies of the immunochemical termination of self-tolerance with unnatural amino acids. Proc. Natl. Acad. Sci. U. S. A. 106, 4337-4342. https://doi.org/10.1073/pnas.0900507106 
Grünewald, J., Tsao, M.L., Perera, R., Dong, L., Niessen, F., Wen, B.G., Kubitz, D.M., Smider, V. V., Ruf, W., Nasoff, M., Lerner, R.A., Schultz, P.G., 2008. Immunochemical termination of self-tolerance. Proc. Natl. Acad. Sci. U. S. A. 105, 11276-11280. https://doi.org/10.1073/pnas.0804157105

Guo, Y.Y., Li, Z.H., Xia, T.Y., Du, Y.L., Mao, X.M., Li, Y.Q., 2019. Molecular mechanism of azoxy bond formation for azoxymycins biosynthesis. Nat. Commun. 10, 1-9. https://doi.org/10.1038/s41467-019-12250-1

Hancock, S.M., Uprety, R., Deiters, A., Chin, J.W., 2010. Expanding the genetic code of yeast for incorporation of diverse unnatural amino acids via a pyrrolysyl-tRNA synthetase/tRNA pair. J. Am. Chem. Soc. 132, 14819-14824. https://doi.org/10.1021/ja104609m

Hayashi, H., Inoue, K., Nagata, T., Kuramitsu, S., Kagamiyama, H., 1993. Escherichia coli Aromatic Amino Acid Aminotransferase: Characterization and Comparison with Aspartate Aminotransferase. Biochemistry 32, 12229-12239. https://doi.org/10.1021/bi00096a036

Hedges, J.B., Ryan, K.S., 2019. In vitro Reconstitution of the Biosynthetic Pathway to the Nitroimidazole Antibiotic Azomycin. Angew. Chemie Int. Ed. 58, 11647-11651. https://doi.org/10.1002/anie.201903500

Hemphill, J., Chou, C., Chin, J.W., Deiters, A., 2013. Genetically encoded light-activated transcription for spatiotemporal control of gene expression and gene silencing in mammalian cells. J. Am. Chem. Soc. 135, 13433-13439. https://doi.org/10.1021/ja4051026

Huynh, K., Hardin, T., Guzman, L., Castillejo, N., Palfey, B., Vey, J., 2020. Flavin binding affinity and initial kinetic characterization of DnmZ, a flavin-dependent $N$-oxygenase. FASEB J. 34, 1-1. https://doi.org/10.1096/fasebj.2020.34.S1.04331 
Indest, K., Eberly, J., Hancock, D., 2015. Expression and characterization of an $N$-oxygenase from Rhodococcus jostii RHAI. J. Gen. Appl. Microbiol 61, 217-223. https://doi.org/10.2323/jgam.61.217

Jackson, J.C., Duffy, S.P., Hess, K.R., Mehl, R.A., 2006. Improving nature's enzyme active site with genetically encoded unnatural amino acids. J. Am. Chem. Soc. 128, 11124-11127. https://doi.org/10.1021/ja061099y

Jenul, C., Sieber, S., Daeppen, C., Mathew, A., Lardi, M., Pessi, G., Hoepfner, D., Neuburger, M., Linden, A., Gademann, K., Eberl, L., 2018. Biosynthesis of fragin is controlled by a novel quorum sensing signal. Nat. Commun. 2018 91 9, 1-13. https://doi.org/10.1038/S41467-018-03690-2

Kim, S., Sung, B.H., Kim, S.C., Lee, H.S., 2018. Genetic incorporation of 1 dihydroxyphenylalanine (DOPA) biosynthesized by a tyrosine phenol-lyase. Chem. Commun. 54, 3002-3005. https://doi.org/10.1039/C8CC00281A

Knoot, C.J., Kovaleva, E.G., Lipscomb, J.D., 2016. Crystal structure of CmlI, the arylamine oxygenase from the chloramphenicol biosynthetic pathway. J Biol Inorg Chem 21, 589603. https://doi.org/10.1007/s00775-016-1363-x

Kretsch, A., M., Morgan, G.L., Tyrrell, J., Mevers, E., Vallet-Gély, I., Li, B., 2018. Discovery of (Dihydro)pyrazine N-Oxides via Genome Mining in Pseudomonas. Org. Lett. 20, 47914795. https://doi.org/10.1021/acs.orglett.8b01944

Kunjapur, A.M., Hyun, J.C., Prather, K.L.J., 2016. Deregulation of S-adenosylmethionine biosynthesis and regeneration improves methylation in the E. coli de novo vanillin biosynthesis pathway. Microb. Cell Fact. 15, 1-17. https://doi.org/10.1186/s12934-016- 
0459-х

617

618

619

620

621

622

623

624

625

626

627

628

629

630

631

632

633

634

635

636

Kunjapur, A.M., Tarasova, Y., Prather, K.L.J., 2014. Synthesis and accumulation of aromatic aldehydes in an engineered strain of Escherichia coli. J. Am. Chem. Soc. 136, 1164411654. https://doi.org/10.1021/ja506664a

Lemke, E.A., Summerer, D., Geierstanger, B.H., Brittain, S.M., Schultz, P.G., 2007. Control of protein phosphorylation with a genetically encoded photocaged amino acid. Nat. Chem. Biol. 3, 769-772. https://doi.org/10.1038/nchembio.2007.44

Lewis, M., Bagwill, C., Hardebeck, L.K.E., Wireduaah, S., 2012. The use of Hammett constants to understand the non-covalent binding of aromatics. Comput. Struct. Biotechnol. J. 1, e201204004. https://doi.org/10.5936/csbj.201204004

Liu, J., Qian, C., Zhang, T., Chen, X., 2009. Study on the synthesis of L-4-nitrophenylalanine. J. Chem. Eng. Chinese Univ. 23(6), 1007-1012.

Lu, H., Chanco, E., Zhao, H., 2012. CmlI is an $N$-oxygenase in the biosynthesis of chloramphenicol. Tetrahedron 68, 7651-7654. https://doi.org/10.1016/j.tet.2012.06.036

Marchand, J.A., Neugebauer, M.E., Ing, M.C., Lin, C.-I., Pelton, J.G., Chang, M.C.Y., 2019. Discovery of a pathway for terminal-alkyne amino acid biosynthesis. Nature 567, 420-424. https://doi.org/10.1038/s41586-019-1020-y

Masuo, S., Zhou, S., Kaneko, T., Takaya, N., 2016. Bacterial fermentation platform for producing artificial aromatic amines. Sci. Rep. 6, 25764. https://doi.org/10.1038/srep25764

Mehl, R.A., Anderson, J.C., Santoro, S.W., Wang, L., Martin, A.B., King, D.S., Horn, D.M., Schultz, P.G., 2003. Generation of a bacterium with a 21 amino acid genetic code. J. Am. 
Chem. Soc. 125, 935-939. https://doi.org/10.1021/ja0284153

638

639

640

641

642

643

644

645

646

647

648

649

650

651

652

653

654

655

656

657

Nepali, K., Lee, H.Y., Liou, J.P., 2019. Nitro-Group-Containing Drugs. J. Med. Chem. 62, 28512893. https://doi.org/10.1021/acs.jmedchem.8b00147

Neumann, H., Hazen, J.L., Weinstein, J., Mehl, R.A., Chin, J.W., 2008. Genetically encoding protein oxidative damage. J. Am. Chem. Soc. 130, 4028-4033. https://doi.org/10.1021/ja710100d

Ng, T.L., Rohac, R., Mitchell, A.J., Boal, A.K., Balskus, E.P., 2019. An N-nitrosating metalloenzyme constructs the pharmacophore of streptozotocin. Nature. https://doi.org/10.1038/s41586-019-0894-z

Nguyen, D.P., Mahesh, M., Elsässer, S.J., Hancock, S.M., Uttamapinant, C., Chin, J.W., 2014. Genetic encoding of photocaged cysteine allows photoactivation of TEV protease in live mammalian cells. J. Am. Chem. Soc. 136, 2240-2243. https://doi.org/10.1021/ja412191m

Nóbile, M.L., Stricker, A.M., Marchesano, L., Iribarren, A.M., Lewkowicz, E.S., 2021. Noxygenation of amino compounds: Early stages in its application to the biocatalyzed preparation of bioactive compounds. Biotechnol. Adv. 51, 107726. https://doi.org/10.1016/j.biotechadv.2021.107726

Onuffer, J.J., Ton, B.T., Klement, I., Kirsch, J.F., 1995. The use of natural and unnatural amino acid substrates to define the substrate specificity differences of Escherichia coli aspartate and tyrosine aminotransferases. Protein Sci. 4, 1743-1749. https://doi.org/10.1002/pro.5560040909

Parker, M.T., Kunjapur, A.M., 2020. Deployment of Engineered Microbes: Contributions to the 
Bioeconomy and Considerations for Biosecurity. Heal. Secur. 18, 278-296. https://doi.org/10.1089/hs.2020.0010

Parry, R., Nishino, S., Spain, J., 2011. Naturally-occurring nitro compounds. Nat. Prod. Rep. 28, 152-167. https://doi.org/10.1039/c0np00024h

Peters, F.B., Brock, A., Wang, J., Schultz, P.G., 2009. Photocleavage of the Polypeptide Backbone by 2-Nitrophenylalanine. Chem. Biol. 16, 148-152. https://doi.org/10.1016/j.chembiol.2009.01.013

Platter, E., Lawson, M., Marsh, C., Sazinsky, M.H., 2011. Characterization of a non-ribosomal peptide synthetase-associated diiron arylamine $N$-oxygenase from Pseudomonas syringae pv. phaseolicola. Arch. Biochem. Biophys. 508, 39-45. https://doi.org/10.1016/j.abb.2011.01.010

Rodriguez, A., Martínez, J.A., Flores, N., Escalante, A., Gosset, G., Bolivar, F., 2014. Engineering Escherichia coli to overproduce aromatic amino acids and derived compounds. Microb. Cell Fact. https://doi.org/10.1186/s12934-014-0126-Z

Schaffer, J.E., Reck, M.R., Prasad, N.K., Wencewicz, T.A., 2017. $\beta$-Lactone formation during product release from a nonribosomal peptide synthetase. Nat. Chem. Biol. 13, 737-744. https://doi.org/10.1038/nchembio.2374

Scott, T.A., Heine, D., Qin, Z., Wilkinson, B., 2017. An L-threonine transaldolase is required for L-threo- $\beta$-hydroxy- $\alpha$-amino acid assembly during obafluorin biosynthesis. Nat. Commun. 8, 15935. https://doi.org/10.1038/ncomms 15935

Seong Choi, Y., Zhang, H., Brunzelle, J.S., Nair, S.K., Zhao, H., 2008. In vitro reconstitution 
and crystal structure of $p$-aminobenzoate $N$-oxygenase (AurF) involved in aureothin biosynthesis. Proc. Natl. Acad. Sci. U. S. A. 105, 6858-6863. https://doi.org/10.1073/pnas.0712073105

Shorter, J., 2009. Electronic Effects of Nitro, Nitroso, Amino and Related Groups, in: PATAI'S Chemistry of Functional Groups. John Wiley \& Sons, Ltd, Chichester, UK. https://doi.org/10.1002/9780470682531.pat0081

Smith, E.E., Linderman, B.Y., Luskin, A.C., Brewer, S.H., 2011. Probing local environments with the infrared probe: L-4-nitrophenylalanine. J. Phys. Chem. B 115, 2380-2385. https://doi.org/10.1021/jp109288j

Stokes, A.L., Miyake-Stoner, S.J., Peeler, J.C., Nguyen, D.P., Hammer, R.P., Mehl, R.A., 2009. Enhancing the utility of unnatural amino acid synthetases by manipulating broad substrate specificity. Mol. Biosyst. 5, 1032-1038. https://doi.org/10.1039/b904032c

Strauss, M.J., 1979. The Nitroaromatic Group in Drug Design. Pharmacology and Toxicology (for Nonpharmacologists). Ind. Eng. Chem. Prod. Res. Dev. 18, 158-166. https://doi.org/10.1021/i360071a002

Sulzbach, M., Kunjapur, A.M., 2020. The Pathway Less Traveled: Engineering Biosynthesis of Nonstandard Functional Groups. Trends Biotechnol. 38, 532-545. https://doi.org/10.1016/j.tibtech.2019.12.014

Thoden, J.B., Branch, M.C., Zimmer, A.L., Bruender, N.A., Holden, H.M., 2013. Active site architecture of a sugar $N$-Oxygenase. Biochemistry 52, 3191-3193. https://doi.org/10.1021/BI400407X 
Tiwari, M.K., Lee, J.K., Moon, H.J., Zhao, H., 2011. Further biochemical studies on aminopyrrolnitrin oxygenase (PrnD). Bioorg. Med. Chem. Lett. 21, 2873-2876. https://doi.org/10.1016/J.BMCL.2011.03.087

Tomita, H., Katsuyama, Y., Minami, H., Ohnishi, Y., 2017. Identification and characterization of a bacterial cytochrome $\mathrm{P} 450$ monooxygenase catalyzing the 3-nitration of tyrosine in rufomycin biosynthesis. J. Biol. Chem. 292, 15859-15869. https://doi.org/10.1074/jbc.M117.791269

Tsao, M.L., Summerer, D., Ryu, Y., Schultz, P.G., 2006. The genetic incorporation of a distance probe into proteins in Escherichia coli. J. Am. Chem. Soc. 128, 4572-4573. https://doi.org/10.1021/ja058262u

Tsutsumi, H., Katsuyama, Y., Izumikawa, M., Takagi, M., Fujie, M., Satoh, N., Shin-ya, K., Ohnishi, Y., 2018. Unprecedented cyclization catalyzed by a cytochrome P450 in benzastatin biosynthesis. J. Am. Chem. Soc. 140, 6631-6639. https://doi.org/10.1021/JACS.8B02769

Völler, J.S., Budisa, N., 2017. Coupling genetic code expansion and metabolic engineering for synthetic cells. Curr. Opin. Biotechnol. 48, 1-7. https://doi.org/10.1016/j.copbio.2017.02.002

Waldman, A.J., Ng, T.L., Wang, P., Balskus, E.P., 2017. Heteroatom-heteroatom bond formation in natural product biosynthesis. Chem. Rev. 117, 5784-5863. https://doi.org/10.1021/acs.chemrev.6b00621

Wang, Y.S., Fang, X., Chen, H.Y., Wu, B., Wang, Z.U., Hilty, C., Liu, W.R., 2013. Genetic incorporation of twelve meta -substituted phenylalanine derivatives using a single 
pyrrolysyl-tRNA synthetase mutant. ACS Chem. Biol. 8, 405-415.

724

725

726

727

728

729

730

731

732

733

734

735

736

737

738

739

740

741

742

Wang, Y.S., Wu, B., Wang, Z., Huang, Y., Wan, W., Russell, W.K., Pai, P.J., Moe, Y.N., Russell, D.H., Liu, W.R., 2010. A genetically encoded photocaged Ne-methyl-1-lysine. Mol. Biosyst. 6, 1557-1560. https://doi.org/10.1039/c002155e

Wannier, T.M., Nyerges, A., Kuchwara, H.M., Czikkely, M., Balogh, D., Filsinger, G.T., Borders, N.C., Gregg, C.J., Lajoie, M.J., Rios, X., Pál, C., Church, G.M., 2020. Improved bacterial recombineering by parallelized protein discovery. Proc. Natl. Acad. Sci. U. S. A. 117, 13689-13698. https://doi.org/10.1073/pnas.2001588117

Welegedara, A.P., Adams, L.A., Huber, T., Graham, B., Otting, G., 2018. Site-specific incorporation of selenocysteine by genetic encoding as a photocaged unnatural amino acid. Bioconjug. Chem. 29, 2257-2264. https://doi.org/10.1021/acs.bioconjchem.8b00254

Wilkins, B.J., Marionni, S., Young, D.D., Liu, J., Wang, Y., Di Salvo, M.L., Deiters, A., Cropp, T.A., 2010. Site-specific incorporation of fluorotyrosines into proteins in Escherichia coli by photochemical disguise. Biochemistry 49, 1557-1559. https://doi.org/10.1021/bi100013s

Winkler, R., Hertweck, C., 2007. Biosynthesis of nitro compounds. ChemBioChem 8, 973-977. https://doi.org/10.1002/cbic.200700042

Winkler, R., Hertweck, C., 2005. Sequential Enzymatic Oxidation of Aminoarenes to Nitroarenes via Hydroxylamines. Angew. Chemie Int. Ed. 44, 4083-4087. https://doi.org/10.1002/ANIE.200500365

Wu, N., Deiters, A., Cropp, T.A., King, D., Schultz, P.G., 2004. A genetically encoded 
photocaged amino acid. J. Am. Chem. Soc. 126, 14306-14307. https://doi.org/10.1021/ja040175z

Yan, G., Yang, M., 2013. Recent advances in the synthesis of aromatic nitro compounds. Org. Biomol. Chem. 11, 2554-2566. https://doi.org/10.1039/c3ob27354g

Yanagisawa, T., Hino, N., Iraha, F., Mukai, T., Sakamoto, K., Yokoyama, S., 2012. Wide-range protein photo-crosslinking achieved by a genetically encoded $\mathrm{N}^{\varepsilon}$-(benzyloxycarbonyl)lysine derivative with a diazirinyl moiety. Mol. Biosyst. 8, 1131-1135. https://doi.org/10.1039/c2mb05321g

Zuo, R., Ding, Y., 2019. Direct Aromatic Nitration System for Synthesis of Nitrotryptophans in Escherichia coli. ACS Synth. Biol. 8, 857-865. https://doi.org/10.1021/acssynbio.8b00534

Zuo, R., Zhang, Y., Jiang, C., Hackett, J.C., Loria, R., Bruner, S.D., Ding, Y., 2017. Engineered P450 biocatalysts show improved activity and regio-promiscuity in aromatic nitration. Sci. Reports 2017 71 7, 1-9. https://doi.org/10.1038/S41598-017-00897-Z

\section{Author Statements}

A.M.K. conceived and supervised the study; N.D.B. designed and performed all experiments, analyzed data, prepared figures, and wrote the manuscript; M.L. aided with molecular cloning; SS cloned the N-oxygenase library and confirmed expression.

\section{Declaration of Competing Interests}

A.M.K. and N.D.B. are co-inventors on a filed patent application related to this technology, and A.M.K. is the owner of this patent.

\section{Acknowledgements}


764 We are grateful to Dr. PapaNii Asare-Okai of the University of Delaware Chemistry Mass 765 Spectrometry Facility for assistance with metabolite LC-MS. We acknowledge support from the 766 following funding sources: The National Science Foundation (NSF CBET \#2032243), University 767 of Delaware Start-Up Funds, the Mort Collins Foundation, and minor research support as part of 768 the Center for Plastics Innovation, an Energy Frontier Research Center funded by the U.S. 769 Department of Energy (DOE), Office of Science, Basic Energy Sciences, under Award No. \# DE770 SC0021166 (mass spectrometry analysis, $N$-oxygenase screening, manuscript preparation). 